\title{
Cholesterol pathway the Achilles heel in prostate cancer metastasis
}

\author{
Isabel Heidegger ${ }^{1}$, Renate Pichler ${ }^{1}$, Andreas Pircher ${ }^{2}$ \\ ${ }^{1}$ Department of Urology, ${ }^{2}$ Department of Internal Medicine V, Hematology and Oncology, Medical University Innsbruck, Innsbruck, Austria \\ Correspondence to: Andreas Pircher, MD, PhD. Associate Professor, Department of Internal Medicine V, Hematology and Oncology, Medical \\ University Innsbruck, Anichstreet 35, 6020 Innsbruck, Austria. Email: andreas.pircher@i-med.ac.at.
}

Submitted Jul 30, 2018. Accepted for publication Aug 09, 2018.

doi: $10.21037 /$ tau.2018.08.12

View this article at: http://dx.doi.org/10.21037/tau.2018.08.12

We read with great interest the research article by Chen et al. (1) published in a previous issue of Nature Genetics as well as the featured article in the "clinical implication for basic research section" of the New England Journal of Medicine by Abate-Shen (2) arguing that the lipogenic program is essential for promotion of metastatic prostate cancer (PC) formation. Chen et al. provide profound genetic data that loss of PTEN and PML induce a sterol regulatory element binding protein (SREBP) dependent lipogenic program leading to formation of more aggressive PC phenotypes. Furthermore the aggressive metastatic tumor phenotype may be dietary induced by intake of high fat diet similar to the western eating habits. Fatostatin, a pharmacological inhibitor of key regulators for fat production (suppression of SREBP pathway) showed potent antitumor and antimetastatic activity in the reported genetic mice models. Summarizing, lipogenesis and external high fat diet are both essential for PC metastasis formation opening new prevention strategies as well as new therapeutic options in PC patients.

Interestingly, the presented findings are complementary to previous studies on primary human PC samples integrating more global/untargeted multi-omics platforms (e.g., unbiased transcriptomics, untargeted metabolomics) showing that PC metastases are essential dependent on metabolic rewiring $(3,4)$. In the presented study by Chen et al the metabolic link was studied exclusively in mice, in addition the authors used transcriptomic profiling together with lipidomics to link genetics and the newly characterized metabolic phenotype (1). Nevertheless, the presented results were not discussed in light of previous published studies $(3,4)$ where Sreekumar et al. (3) could show using untargeted metabolomics that the amino acid sarcosine plays an essential role in PC metastasis formation besides other important metabolites including lipids. Recently, Ylitalo et al. (4) reported that cholesterol biosynthesis, methionine degradation and fatty acid beta-oxidation were the most upregulated pathways in androgen receptor (AR) driven compared to non-AR driven castration-resistant PC bone metastases. In line with the presented findings (1), SREBPs have been reported to be important regulators of cholesterol synthesis and lipogenesis (5). Therefore the SREBP signature shows the ability to link the phenotype observed by Ylitalo et al. (4) and Sreekumar et al. (3) in primary samples with the mouse finding presented here by Chen et al. (1).

The presented results and the already published studies highlight the mechanistic interplay between PC genetics and the underestimated influence of cancer cell metabolism for PC aggressiveness finally leading to the identification of innovative therapeutic targets (e.g., inhibition of cholesterol synthesis, fat restriction). All together this suggests that metabolic rewiring enunciated primary by cholesterol biosynthesis and lipogenesis is a key metabolic target in PC. In addition, we learned from these studies that Omics platforms are complementary $(6,7)$, highlighting the discovery potential of such approaches and their essential impact on translational research.

\section{Acknowledgements}

None.

\section{Footnote}

Conflicts of Interest: The authors have no conflicts of interest 
to declare.

\section{References}

1. Chen M, Zhang J, Sampieri K, et al. An aberrant SREBPdependent lipogenic program promotes metastatic prostate cancer. Nat Genet 2018;50:206-18.

2. Abate-Shen C. Prostate Cancer Metastasis - Fueled by Fat? N Engl J Med 2018;378:1643-5.

3. Sreekumar A, Poisson LM, Rajendiran TM, et al. Metabolomic profiles delineate potential role for sarcosine in prostate cancer progression. Nature 2009;457:910-4.

4. Ylitalo EB, Thysell E, Jernberg E, et al. Subgroups of Castration-resistant Prostate Cancer Bone Metastases Defined Through an Inverse Relationship Between

Cite this article as: Heidegger I, Pichler R, Pircher A. Cholesterol pathway the Achilles heel in prostate cancer metastasis. Transl Androl Urol 2018;7(Suppl 6):S686-S687. doi: 10.21037/tau.2018.08.12
Androgen Receptor Activity and Immune Response. Eur Urol 2017;71:776-87.

5. Horton JD, Goldstein JL, Brown MS. SREBPs: activators of the complete program of cholesterol and fatty acid synthesis in the liver. J Clin Invest 2002;109:1125-31.

6. Goveia J, Pircher A, Conradi LC, et al. Meta-analysis of clinical metabolic profiling studies in cancer: challenges and opportunities. EMBO Mol Med 2016;8:1134-42.

7. Heidegger I, Pichler R, Pircher A. Re: Erik Bovinder Ylitalo, Elin Thysell, Emma Jernberg, et al. Subgroups of Castration-resistant Prostate Cancer Bone Metastases Defined Through an Inverse Relationship Between Androgen Receptor Activity and Immune Response. Eur Urol 2017;71:776-87. Eur Urol 2017;72:e102-e103. 